\title{
Conventional concept of formulations of nanosuspensions
}

\begin{abstract}
Poor water solubility of some of the drug molecules is a major problem that is found to be accountable in drug formulation. So, drug nanosuspensions are found to observe an emerging solution which can be used for safe delivery of such kind of hydrophobic drugs. Scaling down of biocompatible nanoparticles or nanobiomaterials enhances drug aqueous solubility and bioavailability by increasing drug surface area when that comes into contact with biological media. Formulated nanosuspensions are stabilised by various polymers to get effective targeted delivery in cancer tissues and infarct zones with minimal damage to healthy tissues. So, in this review, conventional concept of preparation and application of nanosuspensions are highlighted for achieve effective drug delivery design and their administration routes e.g. parenteral, pulmonary, oral, and ocular which may be helpful for future clinical and pharmaceutical considerations.
\end{abstract}

Keywords: nanosuspensions, nanobiomaterial, nanodelivery, drug delivery, nanoemulsion, parenteral, pulmonary, oral, new chemical entities, biopharmaceutical classification systems
Volume 3 Issue 5 - 2016

Kirti Rani

Amity University Uttar Pradesh, India

Correspondence: Kirti Rani,Assistant Professor (III),Amity Institute of Biotechnology, Amity University Uttar Pradesh, Noida, Sec-125, Gautam Buddha Nagar, Noida-20 I 3 I3 (UP), India,Tel -4392975, Email krsharma@amity.edu

Received: November 10, 2016 | Published: November 18, 2016

\section{Introduction}

Approximately $40 \%$ of new chemical entities (NCE) are lipophilic compounds that have poor aqueous solubility and dissolution which causes their low bioavailability of loaded drug that reaches the systemic circulation. ${ }^{1}$

Therefore, formulating new poorly water soluble molecules are coined to be of two types of molecules called, "Grease ball" and "brick dust" molecules. Grease ball molecules are highly lipophilic as compared to Brick dust molecules, but Brick dust molecules have high melting point above $200^{\circ} \mathrm{C}$ that have strong intermolecular bonding and high lattice energy in solid state. ${ }^{2,3}$

Poorly water soluble molecules are typically formulated for improving their dissolution rate and storage stability by increasing active drug surface area that come in the contact with the dissolution medium. ${ }^{4}$ Most important characteristics of nanosuspensions are their exhibited particle size; polydispersity index, drug saturation solubility, physical stability, dissolution rate and bioavailability that make them more effective drug delivery system as compared to other conventional clinical and pharmaceutical designs. ${ }^{5}$

Their demonstrated advantages are summarised the Biopharmaceutical Classification Systems (BCS) of lipophilic that allocates these drugs further into one of 4 classes: high solubility, high permeability (class I); low solubility and high permeability (class II); high solubility and low permeability (class III); low solubility and low permeability (class IV). ${ }^{6-8}$

Nanofabrication of azithromycin has found to be demonstrated a proven tool to increase its dissolution percentage more than $65 \%$ to get increased drug bioavailability and to achieve the maximum possibility of lipophilic drugs to incorporate them in various nanosuspensions dosage formats such as tablets, pellets, and capsules following standard manufacturing techniques., ${ }^{9,10}$ For example, ketoprofen nanosuspension has been reported that was successfully transformed into pellets with its lower fed/fasted variability. ${ }^{11}$
Previously, "Emulsion Diffusion Method" has been also reported to prepare these kind of drug loaded nanosuspensions by using partially water-miscible and volatile organic solvent such as butyl lactate, benzyl alcohol, triacetin, and ethyl acetate as the dispersed phase systems. ${ }^{12,13}$ These kinds of nanoemulsion formations are prepared by dispersing the drug in a mixture of solvents or an organic solvent by using high pressure homogenisation that lead diffusion of the internal phase into the external phase when droplets convert into semisolid to solid nanoparticles. The only major drawback of this technology was noted to have potential environmental hazards and human safety issues because of using organic solvents such as ethyl acetate, ethanol, methanol, and chloroform. ${ }^{12,14}$

\section{Conclusion}

So, this mini review article can be very useful for depicting the most common conventional methods using various chemical entities which lead to form more effective nanosuspensions which might be used for further advanced clinical therapies and pharmaceutical trials including their high surface area, controllable nanosize dimensions, and tailored surface chemistry. However, these nanosuspensions may have good opportunity for further fabrication of nanoparticles to increase their surface properties to get optimized bioavailability response for treating a number of diseases such as cardiovascular disease, cancer, diabetes, Parkinson's, Alzheimer's. Hence, further studies are also necessary to understand the behaviour of fabricated nanosuspensions in vivo systems, including their interactions with cells and different biological barriers such as the blood-brain barrier and their surface engineering for getting effective active or passive targeting to combat the various clinical challenges that are associated with poorly soluble drugs in order to achieve excellent bioavailability, high dissolution velocity and more potent bioadhesion of the loaded drug to the targeted sites.

\section{Acknowledgements}

I would like to express my cordially appreciation to Amity University Uttar Pradesh, Noida (INDIA). 


\section{Conflicts of interest}

Author declare there are no conflicts of interest.

\section{Funding}

None.

\section{References}

1. Mercadante S, Vitrano V. Pain in patients with lung cancer: pathophysiology and treatment. Lung Cancer. 2010;68(1):10-15.

2. Bhal SK, Kassam K, Peirson IG, et al. The rule of five revisited applying $\log \mathrm{D}$ in place of $\log \mathrm{P}$ in drug-likeness filters. Mol Pharm. 2007;4(4):556-560.

3. Wassvik CM, Holmén AG, Draheim R, et al. Molecular characteristics for solid-state limited solubility. J Med Chem. 2008;51(10):3035-3039.

4. Pifferi G, Restani P. The safety of pharmaceutical excipients. Farmaco. 2007;58(8):541-550.

5. Gao L, Zhang D, Chen M. Drug nanocrystals for the formulation of poorly soluble drugs and its application as a potential drug delivery system. Journal of Nanoparticle Research. 2008;10(5):845-862.

6. Amidon GL, Lennernäs $\mathrm{H}$, Shah VP, et al. A theoretical basis for a biopharmaceutic drug classification: the correlation of in vitro drug product dissolution and in vivo bioavailability. Pharm Res. 1995;12(3):413-420.
7. Kawabata Y, Wada K, Nakatani M, et al. Formulation design for poorly water-soluble drugs based on biopharmaceutics classification system: basic approaches and practical applications. International Journal of Pharmaceutics. 2011;420(1):1-10.

8. Martinez M, Augsburger L, Johnston T, et al. Applying the biopharmaceutics classification system to veterinary pharmaceutical products. Part I: biopharmaceutics and formulation considerations. Adv Drug Deliv Rev. 2002;54(6):805-824.

9. Kesisoglou F, Panmai S, Wu Y. Nanosizing-oral formulation development and biopharmaceutical evaluation. Adv Drug Deliv Rev. 2002;59(7):631-644.

10. Muller RH, Jacobs C, Kayser O. Nanosuspensions as particulate drug formulations in therapy: rationale for development and what we can expect for the future. Adv Drug Deliv Rev. 2001;47(1):3-19.

11. Chaubal MV. Application of formulation technologies in lead candidate selection and optimization. Drug Discov Today. 2004;9(14):603-609.

12. Kocbek P, Baumgartner S, Kristl J. Preparation and evaluation of nanosuspensions for enhancing the dissolution of poorly soluble drugs. Int J Pharm. 2006;312(1-2):179-186.

13. Trotta M, Gallarate M, Pattarino F, et al. Emulsions containing partially water-miscible solvents for the preparation of drug nanosuspensions. $J$ Control Release. 2001;76(1-2):119-128.

14. Dandagi P, Kerur S, Mastiholimath V, et al. Polymeric ocular nanosuspension for controlled release of acyclovir: in vitro release and ocular distribution. Iranian Journal of Pharmaceutical Research. 2009;8(2):79-86. 